\title{
FIELD THEORIES ON CANONICAL AND LIE-ALGEBRA NONCOMMUTATIVE SPACETIMES
}

\section{Giovanni AMELINO-CAMELIA, Michele ARZANO and Luisa DOPLICHER}

\author{
Dipart. Fisica, Univ. Roma "La Sapienza", P.le Moro 2, \\ 00185 Roma, Italy \\ E-mail:amelino@roma1.infn.it,arzano@roma1.infn.it,doplicher@roma1.infn.it
}

\begin{abstract}
Field theories on canonical noncommutative spacetimes, which are being studied also in connection with string theory, and on $\kappa$-Minkowski spacetime, which is a popular example of Lie-algebra noncommutative spacetime, can be naturally constructed by introducing a suitable generating functional for Green functions in energy-momentum space. Direct reference to a star product is not necessary. It is sufficient to make use of the simple properties that the Fourier transform preserves in these spacetimes and establish the rules for products of wave exponentials that are dictated by the non-commutativity of the coordinates. The approach also provides an elementary description of "planar" and "non-planar" Feynman diagrams. We also comment on the rich phenomenology emerging from the analysis of these theories.
\end{abstract}

Noncommutative geometry is being used more and more extensively in attempts to unify general relativity and quantum mechanics. Some "quantum-gravity" approaches explore the possibility that noncommutative geometry might provide the correct fundamental description of spacetime, while in other approaches noncommutative geometry turns out to play a role at the level of the effective theories that describe certain aspects of quantum gravity.

Two simple examples [1] are "canonical noncommutative spacetimes" $(\mu, \nu, \beta=0,1,2,3)$

$$
\left[x_{\mu}, x_{\nu}\right]=i \theta_{\mu \nu}
$$

and "Lie-algebra noncommutative spacetimes"

$$
\left[x_{\mu}, x_{\nu}\right]=i C_{\mu \nu}^{\beta} x_{\beta} .
$$

The canonical type (1) was originally proposed [2] in the context of attempts to develop a new fundamental picture of spacetime. More recently, (1) is proving useful in the description of string theory in certain $B$-field backgrounds (see, e.g., Refs. [3, 因, 5]), with the tensor $\theta_{\mu \nu}$ reflecting the properties of the background.

Among the Lie-algebra spacetimes (2), one of the most studied as an alternative to classical Minkowski spacetime is the $\kappa$-Minkowski [6, ]] spacetime $(l, m=1,2,3)$

$$
\left[x_{m}, t\right]=\frac{i}{\kappa} x_{m}, \quad\left[x_{m}, x_{l}\right]=0 .
$$

One of us recently proposed [8] a new path toward quantum gravity in which $\kappa$-Minkowski would play a key role.

In this paper we aim at giving a short introduction to the construction of field theories in canonical and $\kappa$-Minkowski (Lie-algebra) noncommutative spacetimes, showing that the same strategy can be adopted in both cases.

Building a field theory on these spacetimes will require writing down a generating functional for Green functions that involves products of noncommuting fields. The first observation concerns the way to handle functions of the (noncommuting) spacetime variables. A key point is that one can define such functions as inverse Fourier transforms of some ordinary/commutative energy-momentum-space functions. [. Our functions in noncommutative spacetime will be written as

$$
f(x)=\frac{1}{(2 \pi)^{2}} \int d^{4} k: \exp \left(i k^{\mu} x_{\mu}\right): \tilde{f}(k)
$$

\footnotetext{
${ }^{1}$ Invited talk given by G. Amelino-Camelia at the 25th Johns Hopkins Workshop on Current Problems in Particle Theory, Florence, September 3-5, 2001

${ }^{2}$ The reader will recognize this as a Weyl quantization, [1, 9] which was originally introduced for the noncommuting phase space variables of ordinary quantum mechanics.

${ }^{3}$ Note that, since we always refer to noncommutative spacetime coordinates unless explicitly clarified in the text, in our equations we do not adopt a special notation (such as $\hat{x}$, which is adopted by some authors) for noncommuting spacetime coordinates. Also note that throughout the paper energy-momentum-space coordinates are always commutative.
} 
where $\tilde{f}(k)$ is the Fourier transform of $f$ written in terms of the commuting coordinates and the Fourier parameters $k_{\mu}$ are commuting variables. The function : $\exp \left(i k^{\mu} x_{\mu}\right):$ must be consistent with the properties [10] of the Fourier transform and must reproduce the ordinary $\exp \left(i k^{\mu} x_{\mu}\right)$ in the commutative limit. In canonical spacetimes one can take [1] for : $\exp \left(i k^{\mu} x_{\mu}\right): \operatorname{simply} \exp \left(i k^{\mu} x_{\mu}\right)$ (here of course intended as a function of noncommuting spacetime coordinates and commuting Fourier parameters),

$$
: e^{i k^{\mu} x_{\mu}}:_{\theta} \equiv e^{i k^{\mu} x_{\mu}}
$$

whereas it turns out [11, 12, 10] that in the $\kappa$-Minkowski case the correct formulation of the Fourier theory requires a suitable ordering prescription

$$
: e^{i k^{\mu} x_{\mu}}:_{\kappa} \equiv e^{i k^{m} x_{m}} e^{i k^{0} x_{0}} .
$$

When, in Eq. (4), both $f(x)$ and the phase exponential are ordered as in (6), the function $\tilde{f}(k)$ is again the Fourier transform of $f$ written in terms of the commuting coordinates.

By introducing the Fourier transform one reduces the problem of describing products of fields to the one of establishing the properties of products of exponentials of the noncommuting variables. These can often be evaluated straightforwardly (e.g. using the Baker-Campbell-Hausdorff formula). In the canonical case one finds immediately:

$$
\left(e^{i p^{\mu} x_{\mu}} e^{i k^{\nu} x_{\nu}}\right)_{\theta}=e^{-\frac{i}{2} p^{\mu} \theta_{\mu \nu} k^{\nu}} e^{i(p+k)^{\mu} x_{\mu}},
$$

i.e. the Fourier parameters $p_{\mu}$ and $k_{\mu}$ combine just as usual, with the only new ingredient of an overall phase factor that depends on $\theta_{\mu \nu}$. On the $\kappa$-Minkowski side one introduces ordered exponentials primarily as a way to obtain simple rules for the product of wave exponentials: while wave exponentials of the type $e^{i p^{\mu} x_{\mu}}$ would not combine in a simple way, for the ordered wave exponentials one finds

$$
\left(: e^{i p^{\mu} x_{\mu}}:_{\kappa}\right)\left(: e^{i k^{\nu} x_{\nu}}:_{\kappa}\right)=: e^{i(p \dot{+} k)^{\mu} x_{\mu}}:_{\kappa} \quad .
$$

The notation "+" here introduced reflects the behaviour of the composition of momenta in $\kappa$-Minkowski spacetime. The deformed addition is not symmetric in the two momenta; it works as follows (no sum on repeated indices):

$$
p_{\mu} \dot{+} k_{\mu} \equiv \delta_{\mu, 0}\left(p_{0}+k_{0}\right)+\left(1-\delta_{\mu, 0}\right)\left(p_{\mu}+e^{p_{0} / \kappa} k_{\mu}\right) .
$$

The element $\dot{-} p$ such that $p \dot{+}(\dot{-} p)=0$ is accordingly defined as follows:

$$
(\dot{-} p)_{\mu} \equiv \delta_{\mu, 0}\left(-p_{0}\right)+\left(1-\delta_{\mu, 0}\right)\left(-e^{-p_{0} / \kappa} p_{\mu}\right) .
$$

Readers familiar with the $\kappa$-Poincaré research programme will recognize in (9) the rule for the energy-momentum "coproduct" and in (10) the rule for the "antipode". From (9) one can see that the energy" addition is undeformed while 3-momenta are added according to $\vec{p}+e^{p_{0} / \kappa} \vec{k}$.

In our approach to the construction of these field theories the noncommutativity of the spacetime coordinates is only reflected in the properties of products of wave exponentials, (7) and (8). In similar studies of field theory in noncommutative spacetime one often takes one additional step, by introducing the so called "star-product": the algebra of functions of noncommuting variables is represented by an algebra of ordinary functions endowed with a deformed product. However, when aiming to a formulation of the field theory (Feynman rules) in energymomentum space, one only needs to handle products of exponentials of the coordinates, and there is no need to make explicit reference to a star product (which would allow one to handle general products of functions of the coordinates). The observations (7) and (8) on products of wave exponentials are all one needs (they of course are at the root of the star product, as one can easily realize by appropriate use of Fourier transforms).

From (7) and (8) it is possible to deduce some important features of field theories on a noncommutative spacetime. The noncommutativity of the coordinates of course makes the product of fields noncommutative, and this could be alarming with respect to some of the most significant symmetry properties of Feynman diagrams (such as symmetries under external-line exchange when identical particles are involved). Particularly for $\kappa$ Minkowski it was suspected [11, 12, 13] that this difficulty, reflected in the lack of symmetry of the coproduct (9), would lead to nonsensical results. However, it turns out 14 that this expectation is incorrect. In the

\footnotetext{
${ }^{4}$ There is of course an equally valid alternative ordering prescription in which the time-dependent exponential is placed to the left [12] (while we are here choosing the convention with the time-dependent exponential to the right).

${ }^{5}$ We are here taking the liberty to denominate "energy" the Fourier parameter in the 0-th direction (and similarly for the other three Fourier parameters we use "3-momentum"). This terminology may appear unjustified in the present context, but it is actually meaningful in light of the results on $\kappa$-Minkowski reported in Ref. 11.
} 
following we will show in detail, in the case of a scalar theory with quartic interaction (" $\lambda \Phi^{4}$ theory"), that both in the canonical case and in the $\kappa$-Minkowski case the new features introduced by the noncommutativity do not spoil the desired symmetries under exchange of identical particles.

We start with a generating functional for Green functions (partition function):

$$
\begin{aligned}
Z[J]= & \int \mathcal{D}[\phi] \exp \left(i \int d ^ { 4 } x \left[\frac{1}{2} \partial^{\mu} \phi(x) \partial_{\mu} \phi(x)-\frac{m^{2}}{2} \phi^{2}(x)\right.\right. \\
& \left.\left.-\frac{\lambda}{24} \phi^{4}(x)+\frac{1}{2} J(x) \phi(x)+\frac{1}{2} \phi(x) J(x)\right]\right) .
\end{aligned}
$$

This expression can be maintained in both our examples of noncommutative spacetime. We intend to obtain energy-momentum-space Feynman rules, so we must rewrite the partition function in energy-momentum space, using the representation (仼). We also need an integral representation of the delta function and some elements of the differential calculus in the noncommutative spacetime.

We denote the delta function by $\delta_{D}^{(4)}(k)$, where the subscript $D$ is replaced by $\theta$ on the canonical side and by $\kappa$ on the $\kappa$-Minkowski side. In the canonical case we write:

$$
\delta_{\theta}^{(4)}(k)=\int \frac{d^{4} x}{(2 \pi)^{4}} \exp (i k x)
$$

in very close analogy with the familiar commutative spacetime delta function, while in $\kappa$-Minkowski spacetime we need again an ordered exponential: [12]

$$
\delta_{\kappa}^{(4)}(k)=\int \frac{d^{4} x}{(2 \pi)^{4}}: \exp (i k x):
$$

The manipulations of the partition function on the $\kappa$-Minkowski side will lead to the emergence of terms like $\delta^{(4)}(p \dot{+} k)$. The lack of symmetry of $p \dot{+} k$ then requires some care; the relevant formulas are

$$
\begin{aligned}
\int d^{4} k \delta_{D}^{(4)}(k \dot{+} p) f(k) & =\chi_{1}\left(-p_{0}\right) f(\dot{-} p), \\
\int d^{4} k \delta_{D}^{(4)}(p \dot{+} k) f(k) & =\chi_{2}\left(p_{0}\right) f(\dot{-} p),
\end{aligned}
$$

where $\chi_{1}\left(p_{0}\right)=\chi_{2}\left(p_{0}\right)=1$ in the canonical case and

$$
\begin{aligned}
& \chi_{1}\left(p_{0}\right)=\mu\left(p_{0}\right) \\
& \chi_{2}\left(p_{0}\right)=e^{-3 p_{0} / \kappa} \mu\left(p_{0}\right)
\end{aligned}
$$

for $\kappa$-Minkowski. The function $\mu\left(p_{0}\right)$, which is not needed on the canonical side, is intended [14] to reflect the properties of a non-trivial measure of integration over $\kappa$-energy-momentum space (which, in the sense reflected by (9), is not flat). On $\mu\left(p_{0}\right)$ we will only observe and use the fact that it can depend on energy-momentum only through the 0-th component (energy), 11] postponing to future studies the determination of its exact form. Concerns about the conservation rules have been the most serious obstacle for the construction of physical theories based on $\kappa$-Minkowski, and we shall show that these concerns can be straightforwardly addressed within our approach, independently of the form of $\mu\left(p_{0}\right)$.

On the $\kappa$-Minkowski side one also needs [11] a suitable nontrivial definition of partial derivative:

$$
\begin{aligned}
\frac{\partial}{\partial x_{m}}: e^{i p x}: & =\frac{\partial}{\partial x_{m}} e^{i p x}: \\
\frac{\partial}{\partial x_{0}}: e^{i p x}: & =\kappa:\left(e^{i p x}-e^{i p\left(x+\Delta x_{\kappa}\right)}\right):,
\end{aligned}
$$

where $\left(\Delta x_{\kappa}\right)_{\mu} \equiv-\delta_{\mu, 0} / \kappa$.

\footnotetext{
${ }^{6}$ The careful reader should notice that throughout our analysis formulas for the canonical spacetime are closer to the ones for the familiar case of commutative spacetime. The $\kappa$-Minkowski side requires instead more care. On the canonical side certain simplifications are possible because of the fact that commutators of coordinates are coordinate independent.
} 
Our first objective is to examine the structure of the two-point function at tree-level. For this result we can of course switch off the coupling $\lambda$. Using (14), (15), (18) and (19) one obtains from (11) the (momentum space) deformed partition function of the free theory:

$$
\begin{aligned}
Z_{D}^{0}[J]= & \int \mathcal{D}[\phi] \exp \left(\frac { i } { 2 } \int d ^ { 4 } k \chi _ { 1 } ( k _ { 0 } ) \left[\phi(\dot{-} k)\left[\mathcal{C}_{D}(k)-m^{2}\right] \phi(k)\right.\right. \\
& +J(k) \phi(\dot{-} k)+\phi(k) J(\dot{-} k)]),
\end{aligned}
$$

where in the canonical case the energy-momentum coproduct is trivial, $(\dot{+})_{\theta} \equiv+$, and accordingly also the energy-momentum antipode is trivial, $(\dot{-})_{\theta} \equiv-$. The form of $\chi_{1}\left(k_{0}\right)$ depends on the kind of noncommutativity (as already specified), and $\mathcal{C}_{D}$ represents the mass casimir, i.e. in the canonical case

$$
\mathcal{C}_{\theta}(k)=k_{0}^{2}-\vec{k}^{2}
$$

and in $\kappa$-Minkowski, reflecting the deformed symmetries,

$$
\mathcal{C}_{\kappa}(k)=\kappa^{2}\left(e^{k_{0} / \kappa}+e^{-k_{0} / \kappa}-2\right)-\vec{k}^{2} e^{-k_{0} / \kappa} .
$$

It is convenient to introduce the normalized partition function $\bar{Z}^{0}[J] \equiv Z^{0}[J] / Z^{0}[0]$, and from (20) with simple manipulations one finds that

$$
\bar{Z}_{D}^{0}[J]=\exp \left(-\frac{i}{2} \int d^{4} k \chi_{1}\left(k_{0}\right) \frac{J(k) J(\dot{-} k)}{\mathcal{C}_{D}(k)-m^{2}}\right) .
$$

To obtain Green functions from (23) in the $\kappa$-Minkowski case one also needs appropriately generalized definitions of the functional derivatives:

$$
\begin{gathered}
\frac{\delta F[f]}{\delta f(k)}=\lim _{\varepsilon \rightarrow 0} \frac{1}{\varepsilon}\left(F\left[f(p)+\varepsilon \delta_{D}^{(4)}(p \dot{+}(\dot{-} k))\right]-F[f(p)]\right), \\
\frac{\delta F[f]}{\delta f(\dot{-} k)}=\lim _{\varepsilon \rightarrow 0} \frac{1}{\varepsilon}\left(F\left[f(p)+\varepsilon \delta_{D}^{(4)}(p \dot{+} k)\right]-F[f(p)]\right) .
\end{gathered}
$$

Using (14), (24), (25) and the property $\mathcal{C}_{D}(\dot{-} p)=\mathcal{C}_{D}(p)$, from

$$
G_{0}^{(2)}\left(p, \dot{-} p^{\prime}\right)=-\left.\frac{\delta^{2} \bar{Z}^{0}[J]}{\delta J(\dot{-} p) \delta J\left(p^{\prime}\right)}\right|_{J=0}
$$

one easily obtains the two-point function at tree-level:

$$
G_{0}^{(2)}\left(p, \dot{-} p^{\prime}\right)=\frac{i}{2} \chi_{1}\left(p_{0}\right) \chi_{1}\left(-p_{0}\right) \frac{\delta^{(4)}\left(p \dot{+}\left(\dot{-} p^{\prime}\right)\right)+\delta^{(4)}\left(\left(\dot{-} p^{\prime}\right) \dot{+} p\right)}{\mathcal{C}_{D}(p)-m^{2}}
$$

From the last equation it is easy to verify that in the canonical case the two-point function at tree-level is unmodified. 8 On the $\kappa$-Minkowski side a key role is played by the mass casimir $\mathcal{C}_{\kappa}$, as one might have expected. Importantly, also on the $\kappa$-Minkowski side the two $\delta^{(4)}$ in (27) enforce the same trivial conservation condition; in fact, $\delta^{(4)}\left(\left(\dot{-} p^{\prime}\right) \dot{+} p\right)=e^{3 p_{0} / \kappa} \delta^{(4)}\left(p \dot{+}\left(\dot{-} p^{\prime}\right)\right)=e^{3 p_{0} / \kappa} \delta^{(4)}\left(p-p^{\prime}\right)$. This is a first nontrivial and reassuring result obtained [14] by our approach to field theory in these noncommutative spacetimes: in $\kappa$-Minkowski spacetime we find that, in spite of the nonsymmetric and nonlinear coproduct structure, the usual property that energymomentum is conserved along the tree-level two-point function is maintained.

In order to be able to investigate the properties of the two-point function beyond tree level and in order to establish the form of the tree-level vertex we must now analyze the $O(\lambda)$ contributions to the Green functions. For this we must of course reinstate $\lambda \neq 0$, i.e. we need to analyze $\bar{Z}_{D}^{(1)}[J]$ rather than $\bar{Z}_{D}^{0}[J]$. It turns out to

\footnotetext{
${ }^{7}$ The symmetries of $\kappa$-Minkowski can be described [8] using as key ingredient a $\kappa$-Poincaré Hopf algebra. The relevant $\kappa$-Poincaré mass-casimir relation is $\mathcal{C}_{\kappa}(k)=m^{2}$. The mass parameter $m$, which here appears also in the Lagrangian, is not to be identified with the rest energy $E(\vec{k}=0)$. The rest energy $M$ is obtained from $m$ through the relation $7 m^{2}=\kappa^{2} \sinh ^{2}(M /(2 \kappa))$. (Note that however $m$ and $M$ differ only at order $1 / \kappa^{2}$.)

${ }^{8}$ This could be guessed already at the level of the generating functional, using the fact that the antisymmetry of $\theta_{\mu \nu}$ leads to cyclic dependence of the integrals of product of fields on the order of the fields, and in particular the product of two fields under integral is undeformed.
} 
be useful to rely on some simple relations between $\bar{Z}_{D}^{(1)}[J]$ and $\bar{Z}_{D}^{0}[J]$, which one can obtain with manipulations analogous to the ones described above. For the canonical case one finds

$$
\begin{aligned}
\bar{Z}_{\theta}^{(1)}[J]= & -i \frac{\lambda}{24} \int \delta^{(4)}\left(\sum_{i=1}^{4} k_{i}\right) \prod_{j=1}^{4} \frac{d^{4} k_{j}}{2 \pi} \exp \left(-\frac{i}{2} \sum_{1 \leq i<j \leq 4} k_{i} \times k_{j}\right) . \\
& \frac{\delta}{\delta J\left(-k_{j}\right)} \bar{Z}_{\theta}^{0}[J],
\end{aligned}
$$

where we introduced the notation $p \times q=p^{\mu} \theta_{\mu \nu} q^{\nu}$, while for the $\kappa$-Minkowski case one finds

$$
\bar{Z}_{\kappa}^{(1)}[J]=-i \frac{\lambda}{24} \int \delta^{(4)}\left(\dot{\sum}_{k_{1}, k_{2}, k_{3}, k_{4}}\right) \prod_{j=1}^{4} \frac{d^{4} k_{j}}{2 \pi} \xi\left(k_{j, 0}\right) \frac{\delta}{\delta J\left(\dot{-} k_{j}\right)} \bar{Z}_{\kappa}^{0}[J]
$$

where $\xi\left(k_{j, 0}\right) \equiv 2\left(\mu\left(k_{j, 0}\right)+\mu\left(-k_{j, 0}\right) e^{\frac{3 k_{j, 0}}{\kappa}}\right)^{-1}$, and we introduced a compact ordered-sum notation:

$$
\dot{\sum}_{k_{1}, k_{2}, k_{3}, k_{4}} \equiv k_{1} \dot{+} k_{2} \dot{+} k_{3} \dot{+} k_{4}
$$

The $O(\lambda)$ contribution to the two-point function can be written as

$$
G_{\lambda}^{(2)}\left(p, \dot{-} p^{\prime}\right)=\left(-\left.\frac{\delta^{2} \bar{Z}_{D}^{1}[J]}{\delta J(\dot{-} p) \delta J\left(p^{\prime}\right)}\right|_{J=0}\right)_{\text {connected }} .
$$

In both the canonical and the $\kappa$-deformed case one finds that the 24 connected elements of the one loop deformed two-point function split into two different classes: 16 "planar" contributions and 8 "non-planar" contributions. Planar contributions are associated with the 16 possibilities for attaching the external momenta to consecutive internal lines (e.g. to $k_{1}, k_{2}$ ). The difference between these "planar" diagrams and the diagrams, which can be described as "non-planar", that correspond to the remaining 8 permutations, in which instead the external lines are attached to non-consecutive lines, is meaningful in our noncommutative spacetimes. In fact, on the canonical side the order of the lines coming out of a vertex is reflected in the structure of the $\theta$-dependent phase factors, while on the $\kappa$-Minkowski side the coproduct sum $\dot{\sum}_{k_{1}, k_{2}, k_{3}, k_{4}}$ is not invariant under $k_{1}, k_{2}, k_{3}, k_{4}$ permutations. On the canonical side an example of planar contribution is

$$
\begin{aligned}
& i \frac{\lambda}{24} \int \prod_{j=1}^{4} \frac{d^{4} k_{j}}{2 \pi} \delta^{(4)}\left(\sum_{i=1}^{4} k_{i}\right) \exp \left[-\frac{i}{2}\left(p \times p^{\prime}+2 p \times k_{1}+2 p^{\prime} \times k_{1}\right)\right] . \\
& \left.\left.\left.\cdot \frac{\delta^{2} \bar{Z}_{\theta}^{0}[J]}{\delta J(-p) \delta J\left(-k_{2}\right)}\right|_{J=0} \frac{\delta^{2} \bar{Z}_{\theta}^{0}[J]}{\delta J\left(p^{\prime}\right) \delta J\left(-k_{3}\right)}\right|_{J=0} \frac{\delta^{2} \bar{Z}_{\theta}^{0}[J]}{\delta J\left(-k_{1}\right) \delta J\left(-k_{4}\right)}\right|_{J=0},
\end{aligned}
$$

and an example of non-planar one is

$$
\begin{aligned}
& i \frac{\lambda}{24} \int \prod_{j=1}^{4} \frac{d^{4} k_{j}}{2 \pi} \delta^{(4)}\left(\sum_{i=1}^{4} k_{i}\right) \exp \left[-\frac{i}{2}\left(p \times p^{\prime}+2 p \times k_{1}\right)\right] . \\
& \left.\left.\left.\cdot \frac{\delta^{2} \bar{Z}_{\theta}^{0}[J]}{\delta J(-p) \delta J\left(-k_{2}\right)}\right|_{J=0} \frac{\delta^{2} \bar{Z}_{\theta}^{0}[J]}{\delta J\left(p^{\prime}\right) \delta J\left(-k_{4}\right)}\right|_{J=0} \frac{\delta^{2} \bar{Z}_{\theta}^{0}[J]}{\delta J\left(-k_{1}\right) \delta J\left(-k_{3}\right)}\right|_{J=0} .
\end{aligned}
$$

From (26) and (27) it is possible to see that these expressions contain the term $\delta^{(4)}\left(p-p^{\prime}\right)$, which, in light of the antisymmetry of $\theta_{\mu \nu}$, allows one to ignore terms like $p \times p^{\prime}$ and $2 p \times k_{1}+2 p^{\prime} \times k_{1}$, and leads tot he conclusion that planar terms do not involve any nontrivial $\theta$-dependent phase factors. Nonplanar terms instead do involve nontrivial phase factors of the type $\exp ( \pm i p \times q)$, where $p$ and $q$ represent one external and one internal momentum, and the sign of the exponent depends on the specific momenta involved. Using these observations, and the result on the tree-level two-point function reported above, it is easy to combine all the $O(\lambda)$ (tadpole) contributions (planar and nonplanar) to the full two-point function in the canonical theory. For the truncated two-point function the result is

$$
-i \frac{\lambda}{6} \int \frac{d^{4} k}{(2 \pi)^{4}}(2+\cos (p \times k)) \frac{i}{k^{2}-m^{2}}
$$


where $p$ is the external momentum (the propagation momentum).

In $\kappa$-Minkowski the qualitative (diagrammatic) description of planar and nonplanar contributions is completely analogous, but of course the integrals that are represented by those diagrammatic rules are significantly different. An example of planar contribution is

$$
\begin{aligned}
& i \frac{\lambda}{24} \int \prod_{j=1}^{4} \frac{d^{4} k_{j}}{2 \pi} \xi\left(k_{j, 0}\right) \delta^{(4)}\left(\dot{\sum}_{k_{1}, k_{2}, k_{3}, k_{4}}\right) . \\
& \left.\left.\left.\cdot \frac{\delta^{2} \bar{Z}_{\kappa}^{0}[J]}{\delta J(\dot{-} p) \delta J\left(\dot{-} k_{2}\right)}\right|_{J=0} \frac{\delta^{2} \bar{Z}_{\kappa}^{0}[J]}{\delta J\left(p^{\prime}\right) \delta J\left(\dot{-} k_{3}\right)}\right|_{J=0} \frac{\delta^{2} \bar{Z}_{\kappa}^{0}[J]}{\delta J\left(\dot{-} k_{1}\right) \delta J\left(\dot{-} k_{4}\right)}\right|_{J=0},
\end{aligned}
$$

while an example of non-planar contribution is

$$
\begin{aligned}
& i \frac{\lambda}{24} \int \prod_{j=1}^{4} \frac{d^{4} k_{j}}{2 \pi} \xi\left(k_{j, 0}\right) \delta^{(4)}\left(\dot{\sum}_{k_{1}, k_{2}, k_{3}, k_{4}}\right) . \\
& \left.\left.\left.\cdot \frac{\delta^{2} \bar{Z}_{\kappa}^{0}[J]}{\delta J(\dot{-} p) \delta J\left(\dot{-} k_{2}\right)}\right|_{J=0} \frac{\delta^{2} \bar{Z}_{\kappa}^{0}[J]}{\delta J\left(p^{\prime}\right) \delta J\left(\dot{-} k_{4}\right)}\right|_{J=0} \frac{\delta^{2} \bar{Z}_{\kappa}^{0}[J]}{\delta J\left(\dot{-} k_{1}\right) \delta J\left(\dot{-} k_{3}\right)}\right|_{J=0} .
\end{aligned}
$$

Before looking at how all the planar and all the nonplanar contributions combine in this case to give the $O(\lambda)$ expression of the full two point function in $\kappa$-Minkowski, let us pause on these rather implicit formulas for the $\kappa$-Minkowski tadpole contributions and compare them to the corresponding formulas for the case of canonical spacetimes. One should notice that:

(i) The fact that the tree-level two point functions are different also leads (since the tree-level two-point function appears in the integrand) to significant differences between the $\kappa$-Minkowski case and the canonical case for the computation of the tadpole contributions.

(ii) It is also important that in the canonical case the integration measure is trivial (just as in commutative spacetimes), whereas in the $\kappa$-Minkowski case the measure is nontrivial (and the fact that, at present, there is no consensus on the choice of this measure imposes severe limitations on what can be reliably computed in $\kappa$-Minkowski field theories).

(iii) The spacetime noncommutativity is reflected in energy-momentum space through nontrivial phase factors, on the canonical side, and through nontrivial conservation laws (delta functions) on the $\kappa$-Minkowski side.

(iv) Planar diagrams do not carry any trace of the nontrivial structures mentioned in the previous point. We have already discussed the fact that in the canonical case there is no nontrivial phase factor for the planar contributions, and from (35) one can easily verify [14 that the deformed delta functions of planar tadpole diagrams in $\kappa$-Minkowski combine to ultimately impose trivial/ordinary energy-momentum conservation. [For example, one finds that (35) is proportional to $\delta^{(4)}\left(\dot{-} k_{4} \dot{+}(\dot{-} p) \dot{+} p^{\prime} \dot{+} k_{4}\right) \sim \delta^{(4)}\left(p-p^{\prime}\right)$.]

(v) Nonplanar diagrams encode the most important new features induced by spacetime noncommutativity. We have already discussed the fact that in the canonical case nonplanar contributions do involve nontrivial phase factors, and from (36) one can easily verify [14] that the deformed delta functions of nonplanar tadpole diagrams in $\kappa$-Minkowski combine to ultimately impose deviations from ordinary energymomentum conservation. For example, one finds that (36) is proportional to $\delta^{(4)}\left(\dot{-} k_{3} \dot{+}(\dot{-} p) \dot{+} k_{3} \dot{+} p^{\prime}\right) \sim$ $\delta\left(p_{0}-p_{0}^{\prime}\right) \delta^{(3)}\left(e^{p_{0} / \kappa} \overrightarrow{k_{3}}-\vec{p}+\overrightarrow{k_{3}}+e^{k_{3,0} / \kappa} \overrightarrow{p^{\prime}}\right)$.

These five points (i)-(v) summarize the main differences in the tadpole structure on the two sides, which are representative of the differences that one encounters in the general Feynman-diagram analysis of these theories. It is worth making a few remarks specifically on the deviations from ordinary energy-momentum conservation (in propagation!!) that emerge from the nonplanar tadpole contributions in $\kappa$-Minkowski. In $\kappa$-Minkowski energy conservation is still ordinary also for non-planar diagrams, but momentum conservation is modified and it is modified in a way that cannot even be described as a modified conservation law: the terms involving the loop/integration momenta do not fully cancel each other out in the argument of the leftover (conservation-imposing) delta function. It is easy to verify that these non-planar contributions, while not

\footnotetext{
${ }^{9}$ This should not surprise the readers: the difference between planar and nonplanar diagrams is meaningful because the order of lines attached around a vertex is itself meaningful, and this has its root in the fact that fields do not commute (i.e. it originates directly from noncommutativity).
} 
implementing exactly the ordinary energy-momentum conservation, are still mainly centered around ordinary energy-momentum conservation (assuming reasonably good behaviour at infinity of the expression under the integral). There is therefore some deviation from ordinary conservation of energy-momentum, a sort of fuzzy conservation of momentum, but it is plausible that the full theory (whose construction will also require the integration measure that we are here treating as an unknown) would only predict a very small ( $1 / \kappa$-suppressed) deviation from ordinary conservation of energy-momentum, possibly consistent with observational limits. This issue is here postponed to future studies.

Having noted these main qualitative features of tadpole contributions on the two sides, before moving on to the analysis of interaction vertices, let us note here some formulas that describe the full (truncated) tadpole in $\kappa$-Minkowski. This would be the $\kappa$-Minkowski result that corresponds to the result (34) for the canonical spacetimes. While in the canonical case a large number of simplifications could be exploited to obtain a compact formula, in the $\kappa$-Minkowski case one is stuck with a very long formula which we here split for convenience in two pieces: the sum of the contributions to the truncated tadpole that come from planar diagrams

$$
\begin{aligned}
& i \frac{\lambda}{24} \mu\left(-p_{0}\right) \mu\left(-p_{0}\right) \xi^{2}\left(p_{0}\right) \delta^{(4)}\left(p^{\prime}-p\right) . \\
& \cdot 2\left(1+e^{\frac{p_{0}}{\kappa}}\right)\left(\int \frac{d^{4} k_{4}}{(2 \pi)^{4}} \xi^{2}\left(k_{0,4}\right) \mu^{2}\left(-k_{0,4}\right) \mu\left(k_{0,4}\right) e^{\frac{k_{0,4}}{\kappa}} \frac{e^{-\frac{k_{0,4}}{\kappa}}+1}{\mathcal{C}_{\kappa}\left(k_{4}\right)-m^{2}}+\right. \\
& \left.\sum_{j=1}^{3} \int \frac{d^{4} k_{j}}{(2 \pi)^{4}} \xi^{2}\left(k_{0, j}\right) \mu^{2}\left(-k_{0, j}\right) \mu\left(k_{0, j}\right) \frac{e^{-\frac{k_{0, j}}{\kappa}}+1}{\mathcal{C}_{\kappa}\left(k_{j}\right)-m^{2}}\right),
\end{aligned}
$$

and the sum of the contributions that come from nonplanar diagrams

$$
\begin{aligned}
& i \frac{\lambda}{24} \mu\left(-p_{0}\right) \mu\left(-p_{0}\right) \xi^{2}\left(p_{0}\right) . \\
& \cdot 2\left(\int \frac{d^{4} k_{3}}{(2 \pi)^{4}} \xi^{2}\left(k_{0,3}\right) \mu^{2}\left(-k_{0,3}\right) \mu\left(k_{0,3}\right) \delta^{(4)}\left(\dot{-} k_{3} \dot{+}(\dot{-} p) \dot{+} k_{3} \dot{+} p^{\prime}\right) \frac{e^{-\frac{k_{0,3}}{\kappa}}+1}{\mathcal{C}_{\kappa}\left(k_{3}\right)-m^{2}}+\right. \\
& +\int \frac{d^{4} k_{3}}{(2 \pi)^{4}} \xi^{2}\left(k_{0,3}\right) \mu^{2}\left(-k_{0,3}\right) \mu\left(k_{0,3}\right) \delta^{(4)}\left(\dot{-} k_{3} \dot{+} p^{\prime} \dot{+} k_{3} \dot{+}(\dot{-} p)\right) \frac{e^{-\frac{k_{0,3}}{\kappa}}+1}{\mathcal{C}_{\kappa}\left(k_{3}\right)-m^{2}}+ \\
& +\int \frac{d^{4} k_{2}}{(2 \pi)^{4}} \xi^{2}\left(k_{0,2}\right) \mu^{2}\left(-k_{0,2}\right) \mu\left(k_{0,2}\right) \delta^{(4)}\left(p^{\prime} \dot{+} k_{2} \dot{+}(\dot{-} p) \dot{+}\left(\dot{-} k_{2}\right)\right) \frac{e^{-\frac{k_{0,2}}{\kappa}}+1}{\mathcal{C}_{\kappa}\left(k_{2}\right)-m^{2}}+ \\
& \left.+\int \frac{d^{4} k_{2}}{(2 \pi)^{4}} \xi^{2}\left(k_{0,2}\right) \mu^{2}\left(-k_{0,2}\right) \mu\left(k_{0,2}\right) \delta^{(4)}\left(\dot{-} p \dot{+} k_{2} \dot{+} p^{\prime} \dot{+}\left(\dot{-} k_{2}\right)\right) \frac{e^{-\frac{k_{0,2}}{\kappa}+1}}{\mathcal{C}_{\kappa}\left(k_{2}\right)-m^{2}}\right) .
\end{aligned}
$$

Our next, and final, task is the study of the tree-level vertex. The $O(\lambda)$ contribution to the four-point Green function can be expressed in terms of $\bar{Z}^{(1)}$ through

$$
G_{\lambda}^{(4)}\left(p_{1}, p_{2}, \dot{-} p_{3}, \dot{-} p_{4}\right)=\left.\frac{\delta^{4} \bar{Z}_{D}^{(1)}[J]}{\delta J\left(\dot{-} p_{1}\right) \delta J\left(\dot{-} p_{2}\right) \delta J\left(p_{3}\right) \delta J\left(p_{4}\right)}\right|_{J=0},
$$

and the tree-level (untruncated) vertex is the sum of connected graphs contributing to $G_{\lambda}^{(4)}\left(p_{1}, p_{2}, \dot{-} p_{3}, \dot{-} p_{4}\right)$. The contributions in the two cases we are analyzing are very different. For example one of the contributions ${ }^{10}$ for the canonical case turns out to have the form

$$
-\left.\frac{i \lambda}{24} \int\left(\prod_{l=1}^{4} \frac{d^{4} k_{l}}{2 \pi}\right) \delta^{(4)}\left(\sum_{i=1}^{4} k_{i}\right) \exp \left(-\frac{i}{2} \sum_{1 \leq i<j \leq 4} p_{i} \times p_{j}\right) \frac{\delta^{2} \bar{Z}_{\theta}^{0}[J]}{\delta J\left(-p_{1}\right) \delta J\left(-k_{1}\right)}\right|_{J=0} .
$$

\footnotetext{
${ }^{10}$ The contribution reported in Eq. (40) is actually one of the terms in which the phase factor containing $\theta_{\mu \nu}$ takes its simplest form. In other cases the six terms of type $p_{i} \times p_{j}$ in the exponent have different signs.
} 


$$
\left.\left.\left.\frac{\delta^{2} \bar{Z}_{\theta}^{0}[J]}{\delta J\left(-p_{2}\right) \delta J\left(-k_{2}\right)}\right|_{J=0} \frac{\delta^{2} \bar{Z}_{\theta}^{0}[J]}{\delta J\left(p_{3}\right) \delta J\left(-k_{3}\right)}\right|_{J=0} \frac{\delta^{2} \bar{Z}_{\theta}^{0}[J]}{\delta J\left(p_{4}\right) \delta J\left(-k_{4}\right)}\right|_{J=0}
$$

while the same term in the $\kappa$-Minkowski case takes the form

$$
\begin{aligned}
& -\left.\frac{i \lambda}{24} \int\left(\prod_{l=1}^{4} \frac{d^{4} k_{l}}{2 \pi} \xi\left(k_{l, 0}\right)\right) \delta^{(4)}\left(\dot{\sum}_{k_{1}, k_{2}, k_{3}, k_{4}}\right) \frac{\delta^{2} \bar{Z}_{\kappa}^{0}[J]}{\delta J\left(\dot{-} p_{1}\right) \delta J\left(\dot{-} k_{1}\right)}\right|_{J=0} . \\
& \left.\left.\left.\cdot \frac{\delta^{2} \bar{Z}_{\kappa}^{0}[J]}{\delta J\left(\dot{-} p_{2}\right) \delta J\left(\dot{-} k_{2}\right)}\right|_{J=0} \frac{\delta^{2} \bar{Z}_{\kappa}^{0}[J]}{\delta J\left(p_{3}\right) \delta J\left(\dot{-} k_{3}\right)}\right|_{J=0} \frac{\delta^{2} \bar{Z}_{\kappa}^{0}[J]}{\delta J\left(p_{4}\right) \delta J\left(\dot{-} k_{4}\right)}\right|_{J=0} .
\end{aligned}
$$

We are primarily interested in establishing what are the conservation rules implemented at the vertex. Clearly for the case of canonical noncommutative spacetime one finds the same ordinary energy-momentum conservation rule that applies in the familiar commutative Minkowski spacetime. It is again in the $\kappa$-Minkowski noncommutative spacetime that the most dramatic new features emerge. It is important to observe that (41) is proportional to $\delta^{(4)}\left(\dot{-} p_{1} \dot{+}\left(\dot{-} p_{2}\right) \dot{+} p_{3} \dot{+} p_{4}\right)$ which corresponds to ordinary energy conservation, $-p_{1,0}-p_{2,0}+p_{3,0}+p_{4,0}=0$, but enforces a non-trivial and non-symmetric rule of conservation of 3 -momenta: $-e^{\frac{-p_{1,0}}{\kappa}} \vec{p}_{1}-e^{-\frac{1}{\kappa}\left(p_{1,0}+p_{2,0}\right)} \vec{p}_{2}+$ $e^{-\frac{1}{\kappa}\left(p_{1,0}+p_{2,0}\right)} \vec{p}_{3}+e^{\frac{1}{\kappa}\left(-p_{1,0}-p_{2,0}+p_{3,0}\right)} \vec{p}_{4}=0$. There are 23 other contributions, analogous to (41), to the $\kappa$ Minkowski vertex, and all are proportional to a delta function of the type $\delta^{(4)}\left(\dot{-} p_{1} \dot{+}\left(\dot{-} p_{2}\right) \dot{+} p_{3} \dot{+} p_{4}\right)$ but with different ordering of the momenta $\dot{-} p_{1}, \dot{-} p_{2}, p_{3}, p_{4}$. By using the properties of the coproduct/antipode $(\dot{+} / \dot{-})$ it is easy to see that different ordering possibilities within the argument of the delta function lead to inequivalent conservation rules. This is completely different from the behaviour of the $\kappa \rightarrow \infty$ limit (the limit in which $\kappa$-Minkowski reduces to the ordinary commutative Minkowski spacetime), in which all 24 contributions lead to the same conservation rule $\delta^{(4)}\left(-p_{1}-p_{2}+p_{3}+p_{4}\right)$.

Before making additional remarks it is useful to note here the formulas for the full truncated vertex (putting together the 24 contributions on each side), which in the canonical case takes the form

$$
\begin{gathered}
\frac{-i \lambda}{3} \quad\left(\cos \left(\frac{k_{1} \times k_{4}-k_{2} \times k_{3}}{2}\right)+\cos \left(\frac{k_{1} \times k_{3}+k_{2} \times k_{4}}{2}\right)+\right. \\
\left.+\cos \left(\frac{k_{1} \times k_{2}-k_{3} \times k_{4}}{2}\right)\right) \delta^{(4)}\left(\sum_{i=1}^{4} k_{i}\right),
\end{gathered}
$$

while in $\kappa$-Minkowski it takes the form

$$
\begin{aligned}
& i \frac{\lambda}{24}\left(\frac{i}{2}\right)^{4} \mu\left(-p_{0,1}\right) \mu\left(-p_{0,2}\right) \mu\left(p_{0,3}\right) \mu\left(p_{0,4}\right)\left(\prod_{j=1}^{4} \xi\left(-p_{0, j}\right)\right) . \\
& \cdot\left(\delta^{(4)}\left(\dot{-} p_{1} \dot{+}\left(\dot{-} p_{2}\right) \dot{+} p_{3} \dot{+} p_{4}\right)+\mathcal{P}_{\dot{-} p_{1}, \dot{-} p_{2}, \dot{+} p_{3}, \dot{+} p_{4}}\right),
\end{aligned}
$$

where $\mathcal{P}$ encodes the 24 permutations described above. It is important to notice that, in spite of the lack of symmetry under exchange of momenta that one encounters at intermediate stages of the analysis, the overall structure of the interaction vertices is fully symmetric under exchanges of entering momenta. On the $\kappa$-Minkowski side we are however confronted with a revision of the concept of energy-momentum conservation for scattering processes: since our vertex is not characterized by an overall $\delta$-function (but instead it is split up into different pieces characterized by different $\delta$-function factors), in a given scattering process, with incoming particles characterized by four-momenta $p_{1}$ and $p_{2}$, it becomes impossible to predict the sum of the 3-momenta of the outgoing particles. The theory only predicts that one of our 24 energy-momentum-conservation rules must be satisfied and assigns (equal) probabilities to each of these 24 channels. [1]

We stop here with our analysis of these field theories. Of course, many more issues deserve being discussed: the contributions to the two-point function that, unlike the tadpole, involve a genuine flow of external momenta in loops, the issue of unitarity of the theories, and many other issues. For some of these issues there is not yet a satisfactory analysis in the published literature, and particularly on the $\kappa$-Minkowski side some of

\footnotetext{
${ }^{11}$ These properties of vertices in $\kappa$-Minkowski spacetime represent a rather significant departure from conventional physics and therefore provide a key tool for testing whether Nature makes use of $\kappa$-Minkowski. Making the reasonable assumption [8] that $\kappa$ should be of the order of the Planck scale one easily checks that this prediction for new (non-)conservation rules at the vertex is consistent with all available low-energy data. (In the limit $p_{0} / \kappa \ll 1$ the 24 different conservation rules that characterize our $\kappa$-deformed vertex collapse into a single, and ordinary, conservation rule.)
} 
these issues present us with overwhelming technical and conceptual challenges. 14 There is strong motivation for future studies attempting to address these issues. In fact, these theories in noncommutative spacetimes predict (unlike most "quantum pictures" of spacetime) new physical effects whose magnitude could be large enough for detection (or rejection) in forthcoming experiments. We close by emphasizing some aspects of this phenomenological programme.

It should not go unnoticed that this phenomenology, while certainly interesting, presents us with severe challenges at both the conceptual and quantitative level. Field theories in canonical noncommutative spacetimes host a highly nontrivial infrared structure. Some evidence of this can be seen by calculating the integral in Eq. (34) and realizing that the result has singular behaviour at small external momentum. More careful discussions of this feature (and of its origin in the so-called "IR/UV mixing") can be found, e.g., in Refs. 四, 15. 16]. The nontrivial infrared structure poses a severe challenge [16, 17] to our conventional way to do phenomenology (but unfortunately this challenge has been largely underestimated in the large majority of publications on this subject).

These infrared problems of the phenomenology on the canonical side do not appear to be present on the $\kappa$-Minkowski side. However, there one is confronted with the fact that the natural estimate of $\kappa$ as the Planck scale 8] (or somewhere in that neighborhood) leads to the prediction of very small effects. It is natural to identify $\kappa$ with a fundamental/meaningful scale since $\kappa$ is an invariant of the appropriate formulation [8] of Lorentz transformations that applies in $\kappa$-Minkowski (different inertial observers attribute the same numerical value to $\kappa$ ). For the entries in the $\theta$ matrix on the canonical side there is instead no natural estimate, since their values are observer-dependent (the proper concept of Lorentz transformations in canonical noncommutative spacetime is just the ordinary one and under those Lorentz transformations $\theta$ behaves like a tensor, i.e. its components take different numerical values for different inertial observers).

Perhaps the most exciting phenomenological developments in this area would come if (at least in these elementary quantum spacetimes) one could give a satisfactory description [18] of spacetime fuzziness. Experimental sensitivity to certain possible manifestations of fuzziness is improving very quickly. However, our present understanding of fuzziness in these spacetimes is still very limited. 19

At least for the short-term future a more promising opportunity for experimental tests comes from the emergence of deformed dispersion relations in these noncommutative spacetimes. We have shown above that the dispersion relation is modified already at tree level in $\kappa$-Minkowski. On the canonical side the tree-level dispersion relation is undeformed, but loop effects introduce severe deformation. [Again one can get a first glimpse at this feature by calculating the integral in Eq. (34).] Experimental sensitivities to "in vacuo dispersion", 20, 21 a characteristic signature of deformed dispersion relations, have improved dramatically in recent times. In particular, within a few years the $\kappa$-Minkowski dispersion relation will be tested [21, 22, 23] with sensitivity all the way up to $\kappa$ of order the Planck scale.

Deformed dispersion relations are also being analyzed in relation with the emergence of deformed threshold conditions for particle-production processes, [24, 25, 26, 27] another prediction which could be tested with remarkable sensitivity. 28, 29]

\section{References}

[1] J. Madore, S. Schraml, P. Schupp and J. Wess, Eur. Phys. J. C 16, 161 (2000).

[2] S. Doplicher, K. Fredenhagen and J.E. Roberts, Phys. Lett. B 331, 39 (1994).

[3] N. Seiberg and E. Witten, JHEP 9909, 032 (1999).

[4] A. Matusis, L. Susskind and N. Toumbas, JHEP 0012, 002 (2000).

[5] Chong-Sun Chu, B.R. Greene and G. Shiu, Mod. Phys. Lett. A 16, 2231 (2001).

[6] S. Majid and H. Ruegg, Phys. Lett. B 334, 348 (1994).

[7] J. Lukierski, H. Ruegg and W.J. Zakrzewski Ann. Phys. 243, 90 (1995).

[8] G. Amelino-Camelia, gr-qc/0012051, Int. J. Mod. Phys. D 11, 35 (2002); hep-th/0012238, Phys. Lett. B 510, 255 (2001).

[9] L. Castellani, Class. Quant. Grav. 17, 3377 (2000).

[10] S. Majid and R. Oeckl, Commun. Math. Phys. 205, 617(1999).

[11] G. Amelino-Camelia and S. Majid, Int. J. Mod. Phys. A 15, 4301 (2000). 
[12] P. Kosinski, J. Lukierski and P. Maslanka, Czech. J. Phys. 50, 1283 (2000).

[13] P. Kosinski, J. Lukierski and P. Maslanka Phys. Rev. D 62, 025004 (2000).

[14] G. Amelino-Camelia and M. Arzano, hep-th/0105120, Phys. Rev. D (in press).

[15] S. Minwalla, M. Van Raamsdonk and N. Seiberg, JHEP 0002, 020 (2000).

[16] G. Amelino-Camelia, L. Doplicher, S. Nam and Y. Seo, hep-th/0109191.

[17] G. Amelino-Camelia, G. Mandanici and K. Yoshida, in preparation.

[18] G. Amelino-Camelia, gr-qc/9808029, Nature 398, 216 (1999); gr-qc/0104005.

[19] G. Amelino-Camelia, Phys. Lett. B 392, 283 (1997).

[20] G. Amelino-Camelia, J. Ellis, N.E. Mavromatos and D.V. Nanopoulos, Int. J. Mod. Phys. A12, 607 (1997).

[21] G. Amelino-Camelia, J. Ellis, N.E. Mavromatos, D.V. Nanopoulos and S. Sarkar, astro-ph/9712103, Nature 393, 763 (1998).

[22] S. D. Biller et al, Phys. Rev. Lett. 83, 2108 (1999).

[23] J.P. Norris, J.T. Bonnell, G.F. Marani and J.D. Scargle, astro-ph/9912136; A. de Angelis, astro$\mathrm{ph} / 0009271$.

[24] T. Kifune, Astrophys. J. Lett. 518, L21 (1999).

[25] R.J. Protheroe and H. Meyer, Phys. Lett. B 493, 1 (2000).

[26] G. Amelino-Camelia and T. Piran, Phys. Lett. B 497, 265 (2001); Phys. Rev. D 64, 036005 (2001).

[27] T. Jacobson, S. Liberati and D. Mattingly, hep-ph/0112207.

[28] M. Takeda et al, Phys. Rev. Lett. 81, 1163 (1998).

[29] F.A. Aharonian et al, A\&BA 349, 11A (1999). 